\title{
Convexity of harmonic densities
}

\author{
David Benko, Peter Dragnev and Vilmos Totik
}

\begin{abstract}
The convexity of the densities of harmonic measures is proven for subsets of a circle or of the real line. As a consequence, we get the convexity of the densities of equilibrium measures for compact sets lying on circles or the real axis.
\end{abstract}

\section{Introduction and results}

Equilibrium measures, Green's functions, balayage measures and harmonic measures are basic objects of potential theory. There are thousands of papers on them with an enormous number of connections and applications. In this paper we establish a basic convexity property of these quantities for sets lying on the real line or on a circle. The predecessor of this work was [2], where the results below were proven for the case when $F$ is one or two intervals/arcs. An extension to Riesz kernels, as well as applications of the convexity results to external field problems and constrained energy problems, are presented in the forthcoming paper [3].

We refer to [4] or [6] for the basic concepts of logarithmic potential theory. All the measures below will be finite Borel measures. If $G$ is a domain, $E \subseteq \partial G$ is a closed set and $\lambda \in G$, then $\omega(\lambda, E ; G)$ denotes the harmonic measure of $E$ at $\lambda$ with respect to $G$.

A positive function on an interval is called log-convex if its logarithm is a convex function. This is stronger than mere convexity, and the product of log-convex functions is clearly log-convex. We shall also need that the sum of log-convex functions is also log-convex: log-convexity of $f$ means continuity and the inequality

$$
f\left(\frac{x+y}{2}\right) \leq \sqrt{f(x) f(y)}
$$

and if we know this for $f$ and $g$ then it also follows for $f+g$ since then

$$
(f+g)\left(\frac{x+y}{2}\right) \leq \sqrt{f(x) f(y)}+\sqrt{g(x) g(y)} \leq \sqrt{(f+g)(x)(f+g)(y)},
$$

Mathematics Subject Classification (2010): 31A15.

Keywords: Convexity, harmonic measures, equilibrium measures, balayage. 
where the last inequality follows from the arithmetic-geometric mean inequality after squaring both sides.

Our main results are:

Theorem 1.1. If $F \subset \mathbb{R}$ is a closed set, $\lambda \in \mathbb{R} \backslash F$ and $I \subset F$ is an interval, then the density of the harmonic measure $\omega(\lambda, \cdot ; \overline{\mathbb{C}} \backslash F)$ with respect to Lebesgue measure on $\mathbb{R}$ is log-convex on $I$.

Theorem 1.2. Let $C$ be a circle on the plane. If $F \subset C$ is a closed set, $\lambda \in C \backslash F$ and $I \subset F$ is an arc, then the density of the harmonic measure $\omega(\lambda, \cdot ; \overline{\mathbb{C}} \backslash F)$ with respect to arc measure on $C$ is log-convex on $I$.

In both theorems the harmonic measures are absolutely continuous on $I$ (see Lemma 3.1), so the densities in question exist.

Theorem 1.1 is a limit case of Theorem 1.2 when the radius of the circle tends to infinity, but because of its importance we have separated it. The proofs in both cases have the same ideas.

We also mention that even though circles are images of the real line under Möbius transformations, Theorem 1.2 does not seem to be a transformed case of Theorem 1.1, since Möbius transformations do not preserve convexity.

We shall prove Theorems 1.1-1.2 in the following equivalent form. Denote by $\operatorname{Bal}(\rho, F)$ the balayage of a measure $\rho$ (with $\rho(F)=0$ ) onto $F$ (often said "out of $\left.\overline{\mathbb{C}} \backslash F^{\prime \prime}\right)$. See Chapter IV of [5] or Section II.4 of [8] for a detailed introduction to balayage measures and their properties. In particular, the balayage measures in our discussion vanish on sets of zero capacity, and then they are unique (see Theorem 4.6 in [5]).

Theorem 1.3. If $F \subset \mathbb{R}$ is a closed set, $\rho$ is a measure on $\mathbb{R} \backslash F$ and $I \subset F$ is an interval, then the density of $\operatorname{Bal}(\rho, F)$ with respect to Lebesgue measure on $\mathbb{R}$ is log-convex on $I$.

Theorem 1.4. Let $C$ be a circle on the plane. If $F \subset C$ is a closed set, $\rho$ is a measure on $C \backslash F$ and $I \subset F$ is an arc, then the density of $\operatorname{Bal}(\rho, F)$ with respect to arc measure on $C$ is log-convex on $I$.

In fact, if $\delta_{\lambda}$ denotes the Dirac delta at $\lambda$, then $\omega(\lambda, \cdot ; \overline{\mathbb{C}} \backslash F)$ is just $\operatorname{Bal}\left(\delta_{\lambda}, F\right)$ :

$$
\omega(\lambda, E ; \overline{\mathbb{C}} \backslash F)=\operatorname{Bal}\left(\delta_{\lambda}, F\right)(E),
$$

for all Borel set $E \subset F$ (see, e.g., (A.3.3) in [8]), so Theorem 1.1 is the $\rho=\delta_{\lambda}$ special case of Theorem 1.3. Conversely,

$$
\operatorname{Bal}(\rho, F)=\int \operatorname{Bal}\left(\delta_{\lambda}, F\right) d \rho(\lambda)=\int \omega(\lambda, \cdot ; \overline{\mathbb{C}} \backslash F) d \rho(\lambda),
$$

and hence Theorem 1.3 is an easy consequence of Theorem 1.1. The same can be said of Theorems 1.2 and 1.4 . 


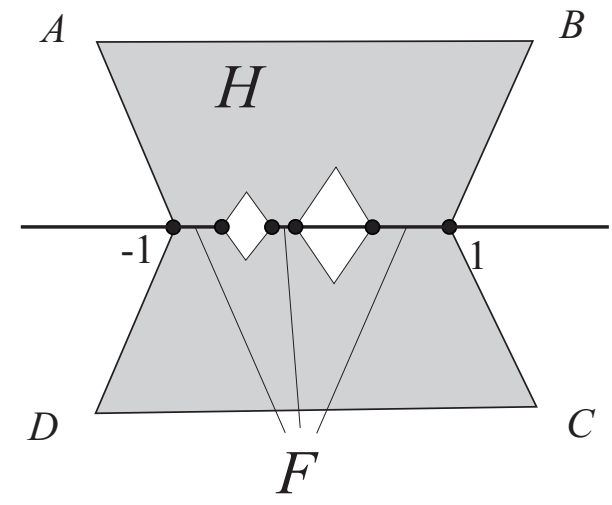

Figure 1. The set $H$, where the vertices $A, B, C$ and $D$ are the points $( \pm(1+$ $\sqrt{2 / 3}), \pm \sqrt{2}$ ), and the white rhomboids with side slopes $\pm \sqrt{3}$ are erected above the subintervals of $[-1,1] \backslash F$.

For later use note also the following consequence of (1.2): if $u$ is a continuous function on $\overline{\mathbb{C}}$ which is harmonic in $\mathbb{C} \backslash F$, then

$$
\int u d \operatorname{Bal}(\rho, F)=\int u d \rho
$$

(see also Theorems II.4.1 and II.4.4 in [8]). As an immediate consequence we obtain:

Theorem 1.5. If $F \subset \mathbb{R}$ or $F \subset C$ as in Theorems 1.1-1.2 is compact, then the equilibrium measure of $F$ has log-convex density on any subinterval of $F$.

Indeed, for $F \subset \mathbb{R}$ this is just the $\lambda=\infty$ (or $\lambda \rightarrow \infty)$ special case of Theorem 1.1 (see Theorem 4.3.14 in [6]). For $F \subset C$ the theorem follows from Theorem 1.4, since the equilibrium measure is nothing else than the balayage of the normalized arc measure on $C$ onto $F$.

The theorems above imply the convexity of harmonic densities on a considerably larger set than what is stated in those theorems. Consider for example the case of the real line and assume that $F$ consists of finitely many intervals. We may also assume that $F \subset[-1,1]$ and $\pm 1 \in F$. Consider the open set $H$ depicted in Figure 1, where the horizontal line segments are at height $\pm \sqrt{2}$ and all other line segments have slope $\pm \sqrt{3}$.

Corollary 1.6. With these notations, for all $\lambda \in \overline{\mathbb{C}} \backslash H$ the density of the harmonic measure $\omega(\lambda, \cdot ; \overline{\mathbb{C}} \backslash F)$ is convex on every subinterval of $F$.

For log-convexity the exceptional region $H$ would be slightly larger: the slopes of the corresponding slanted lines would be \pm 1 instead of $\pm \sqrt{3}$.

Below we make an observation regarding Green's functions. For a domain $G \subset \mathbb{C}$ whose boundary is the union of $C^{2}$-smooth Jordan curves and for a 
point $\lambda \in G$, let $g_{G}(z, \lambda)$ denote the Green's function in $G$ with pole at $\lambda$. Then (see Theorem II.4.11 in [8]) we have on the boundary of $G$ the formula

$$
d \operatorname{Bal}\left(\delta_{\lambda}, \partial G\right)=\frac{1}{2 \pi} \frac{\partial g_{G}(s, \lambda)}{\partial \mathbf{n}} d s
$$

where $d s$ is arc-length measure and $\mathbf{n}$ denotes the inner normal to $G$. By applying a standard limiting process, we can derive the following: if $F \subset \mathbb{R}$ consists of finitely many closed intervals and $\lambda \in \mathbb{R}$ is a point outside $F$, then for $x$ lying inside $F$,

$$
\frac{d \operatorname{Bal}\left(\delta_{\lambda}, F\right)}{d x}=\frac{1}{2 \pi}\left(\frac{\partial g_{\overline{\mathbb{C}} \backslash F}(x, \lambda)}{\partial \mathbf{n}_{+}}+\frac{\partial g_{\overline{\mathbb{C}} \backslash F}(x, \lambda)}{\partial \mathbf{n}_{-}}\right)=\frac{1}{\pi} \frac{\partial g_{\overline{\mathbb{C}} \backslash F}(x, \lambda)}{\partial \mathbf{n}_{+}},
$$

where $\mathbf{n}_{ \pm}$denote the two normals to the real line at $x$ and in the last step we used the symmetry of the Green's function $g_{\overline{\mathbb{C}} \backslash F}(., \lambda)$. Therefore (since we shall prove strict log-convexity in our theorems), it follows from Theorem 1.3 that if $I$ is a closed interval lying in the (one-dimensional) interior of $F$, then for sufficiently small $\tau>0$ the function $g_{\mathbb{C} \backslash F}(x+i \tau, \lambda)$ (with real $\lambda$ ) is convex on $I$. This can be translated into a statement about the level curves $L_{\delta}=\left\{z: g_{\overline{\mathbb{C}} \backslash F}(z, \lambda)=\delta\right\}$ of the Green's function: for small $\delta>0$, the portion of this level curve lying above $I$ is horizontally convex (meaning that the curve lies above its horizontal chords). Note however, that this level curve need not be convex even if $F$ consists of a single interval, say $F=[-1,1]$ : one can easily derive from formula (2.1) below that if $\lambda>1$ is close to 1 , then the reciprocal of $\partial g_{\overline{\mathbb{C}} \backslash F}(x, \lambda) / \partial \mathbf{n}$ is not a concave function on the interval $[8 / 10,9 / 10]$ and hence the $\delta$-level curve of $g_{\overline{\mathbb{C} \backslash[-1,1]}}(z, \lambda)$ for sufficiently small $\delta>0$ is not convex in the sense that over the interval $[8 / 10,9 / 10]$ the curve lies below its chords.

The following section contains the proofs of Theorems 1.3 and 1.4. The last section contains four simple lemmas on balayage measures and their convergence which we need in the proofs.

\section{Proofs}

Proof of Theorem 1.3. Case I. F is an interval. It is sufficient to prove the result for $\rho=\delta_{\lambda}$, where $\lambda \notin F$ (see (1.2)). If $F=[a, b]$, then the density in question is (see (II.4.47) in [8])

$$
\frac{d \operatorname{Bal}\left(\delta_{\lambda}, F\right)}{d x}=\frac{1}{\pi} \frac{1}{|\lambda-x|} \frac{\sqrt{|\lambda-a||\lambda-b|}}{\sqrt{|x-a||x-b|}},
$$

and this is clearly log-convex.

The $a=-\infty$ or $b=\infty$ cases can be obtained from this by letting $a \rightarrow-\infty$, or $b \rightarrow \infty$.

For later reference let us also mention that the density of the balayage of $\delta_{\lambda}$ onto the complement of the finite interval $(a, b)$ is given by the same formula $(2.1)$ (just in this case $x \in \mathbb{R} \backslash[a, b]$ while in $(2.1)$ we have $x \in(a, b)$ ). See Lemma 2.3 
in [9] or apply the transformation $x \rightarrow(x-(a+b) / 2)^{-1}$ which maps $\mathbb{R} \backslash(a, b)$ into $[A, B]=[-2 /(b-a), 2 /(b-a)]$, use that harmonic measures (hence balayages of point masses) are conformally invariant, and apply formula (2.1) to $[A, B]$ (the calculations are simple if $[a, b]=[-1,1]$, which can be assumed). Therefore, this "one interval case" also covers the situation where $F=(-\infty, a] \cup[b, \infty)$ is the union of two intervals "joined" at $\infty$ (and hence considered as one).

Case II. F consists of finitely many intervals. First we prove the following lemma, in which $\|\rho\|=\rho(\overline{\mathbb{C}})$ denotes the total mass of the measure $\rho$.

Lemma 2.1. Let $F$ consist of finitely many intervals and let $I \subset F$ be a subinterval of $F$. Suppose that there is an $\alpha<1$ for which the following is true: for every $\rho$ with $\rho(F)=0$ there are measures $\nu$ and $\mu$ such that $\nu$ is supported on $F$, it has log-convex density on $I, \mu(F)=0,\|\mu\| \leq \alpha\|\rho\|$ and $\operatorname{Bal}(\rho, F)=\nu+\operatorname{Bal}(\mu, F)$. Then for all measures $\rho$ with $\rho(F)=0$ the density of $\operatorname{Bal}(\rho, F)$ is log-convex on $I$.

Proof. Indeed, let $\nu_{1}=\nu, \mu_{1}=\mu$ and apply the assumption with $\rho=\mu_{1}$. There are $\nu_{2}, \mu_{2}$ such that $\nu_{2}$ is supported on $F$, it has log-convex density on $I, \mu_{2}(F)=0$, $\left\|\mu_{2}\right\| \leq \alpha\left\|\mu_{1}\right\| \leq \alpha^{2}\|\rho\|$, and $\operatorname{Bal}\left(\mu_{1}, F\right)=\nu_{2}+\operatorname{Bal}\left(\mu_{2}, F\right)$, i.e., $\operatorname{Bal}(\rho, F)=\nu_{1}+\nu_{2}$ $+\operatorname{Bal}\left(\mu_{2}, F\right)$. Iterating this process we get measures $\nu_{k}, \mu_{k}$ with similar properties such that

$$
\operatorname{Bal}(\rho, F)=\nu_{1}+\nu_{2}+\cdots+\nu_{k}+\operatorname{Bal}\left(\mu_{k}, F\right) ; \quad\left\|\mu_{k}\right\| \leq \alpha^{k}\|\rho\| .
$$

As $\mu_{k} \rightarrow 0$ in the weak* topology when $k \rightarrow \infty$, Lemma 3.2 gives that if $v$ denotes the density of $\operatorname{Bal}(\rho, F)$ and $v_{k}$ denotes the density of $\nu_{k}$ on $I$, then

$$
v=v_{1}+v_{2}+\cdots,
$$

where the series converges uniformly on compact subsets of the interior of $I$, and the conclusion follows.

Now we return to the proof of Theorem 1.3. Thus, let $F=\bigcup_{i=1}^{m} I_{i}$ consist of finitely many intervals $I_{i}$. Without loss of generality we may assume that $F$ is compact, for if one or two of the $I_{i}$ 's is infinite, then we simply consider the compact sets $F \cap[-L, L]$ and let $L$ tend to infinity (cf. Lemma 3.3). Now let $\mathbb{R} \backslash F=\bigcup_{j=1}^{m} J_{j}$ be the decomposition of the complement of $F$ into its subintervals with the understanding that the two infinite subintervals in the complement are considered as one (of the type $(-\infty, a] \cup[b, \infty)$, "joined" at $\infty$ ). Choose a $\delta>0$ smaller than the length of the shortest $I_{i}$. We claim that there is a $c_{\delta}>0$ such that if $\rho_{j}$ is a measure on one of the $J_{j}$ 's, then $\operatorname{Bal}\left(\rho_{j}, \mathbb{R} \backslash J_{j}\right)(F) \geq c_{\delta}\left\|\rho_{j}\right\|$, i.e., at least $c_{\delta}\left\|\rho_{j}\right\|$ mass of the measure $\operatorname{Bal}\left(\rho_{j}, \mathbb{R} \backslash J_{j}\right)$ is supported on $F$. In fact, if $J_{j}=[a, b]$ is finite then it is clear from (1.2) and (2.1) (recall that (2.1) is still the density of $\operatorname{Bal}\left(\delta_{\lambda}, \mathbb{R} \backslash J_{j}\right)$ in this case) that

$$
\operatorname{Bal}\left(\rho_{j}, \mathbb{R} \backslash J_{j}\right)([a-\delta, a] \cup[b, b+\delta]) \geq c_{\delta}\left\|\rho_{j}\right\|,
$$

and notice that $[a-\delta, a] \cup[b, b+\delta] \subseteq F$. When $J_{j}$ is infinite, say $(-\infty, a) \cup(b, \infty)$, the argument is similar. 
Now if $\rho$ is any measure on $\mathbb{R}$ with $\rho(F)=0$ then select a $j$ such that $\rho\left(J_{j}\right) \geq$ $\|\rho\| / m$ and with $\rho_{j}=\rho_{I_{j}}$ let

$$
\nu=\left.\operatorname{Bal}\left(\rho_{j}, \mathbb{R} \backslash J_{j}\right)\right|_{F}
$$

be the restriction to $F$ of the balayage of $\rho_{j}$ onto $\mathbb{R} \backslash J_{j}$ and let

$$
\mu=\left.\operatorname{Bal}\left(\rho_{j}, \mathbb{R} \backslash J_{j}\right)\right|_{\mathbb{R} \backslash F}+\rho_{\mid \mathbb{R} \backslash J_{j}}
$$

be the rest of this balayage plus the rest of $\rho$. It is clear that $\nu$ is supported on $F$, it has log-convex density on any subinterval of $F$ by the one interval case (Case I) verified above, and, as we have just seen, $\|\nu\| \geq c_{\delta}\|\rho\| / m$. Measure $\mu$ is carried by $\mathbb{R} \backslash F$ (i.e., $\mu(F)=0$ ) and, according to what we have just said, $\|\mu\| \leq\|\rho\|-\|\nu\| \leq\left(1-c_{\delta} / m\right)\|\rho\|$. Finally, the balayage of $\rho_{j}$ on $F$ can be obtained in two steps: first take the balayage of $\rho_{j}$ onto $\mathbb{R} \backslash J_{j}$, and then take the balayage of that onto $F$, i.e.,

$$
\operatorname{Bal}\left(\rho_{j}, F\right)=\left.\operatorname{Bal}\left(\rho_{j}, \mathbb{R} \backslash J_{j}\right)\right|_{F}+\operatorname{Bal}\left(\left.\operatorname{Bal}\left(\rho_{j}, \mathbb{R} \backslash J_{j}\right)\right|_{\mathbb{R} \backslash F}, F\right),
$$

which shows that $\operatorname{Bal}(\rho, F)=\nu+\operatorname{Bal}(\mu, F)$. This proves that with $\alpha=1-c_{\delta} / m$ the assumptions in Lemma 2.1 are satisfied, therefore the claim in the theorem follows from Lemma 2.1.

Case III. $\overline{\mathbb{C}} \backslash F$ is regular with respect to the Dirichlet problem. First, Let $F$ be compact, and let $F_{n}$ be the set of points in $\mathbb{R}$ the distance of which to $F$ is at most $1 / n$. Then $F=\bigcap_{n} F_{n}, F_{n+1} \subset F_{n}, F_{n}$ consists of finitely many intervals, and if $\rho(F)=0$, then $\rho_{\mathbb{R}_{\mathbb{R} \backslash F_{n}}} \rightarrow \rho$ in the weak* topology. Therefore, by Lemma 3.2, the densities of $\operatorname{Bal}\left(\rho_{\mathbb{R} \backslash F_{n}}, F_{n}\right)$ tend uniformly to $\operatorname{Bal}(\rho, F)$ on compact subsets of the interior of $I$. Since the former are all log-convex on $I$ by Case II, the log-convexity of the density of $\operatorname{Bal}(\rho, F)$ on $I$ follows.

If $F$ is unbounded, then apply what we have just proven to some appropriate $F^{m}=F \cap\left[L_{m}, M_{m}\right]$, where $L_{m} \rightarrow-\infty$ and $M_{m} \rightarrow \infty$, for which $\overline{\mathbb{C}} \backslash F^{m}$ is regular (say $L_{m} \in \mathbb{R} \backslash F$ if $R$ does not contain an infinite interval $(-\infty, a)$ and $L_{m} \in(-\infty, a)$ if $\left.(-\infty, a] \subseteq F\right)$ and take the limit $m \rightarrow \infty$ as before.

Case IV. F is arbitrary. By Ancona's theorem [1] for every $n$ there is a regular set $F_{n} \subset F$ for which the capacity of $F \backslash F_{n}$ is smaller than $1 / n$. Since the union of regular sets is regular, we may assume $I \subseteq F_{n} \subseteq F_{n+1}$ for all $n$. Now we can invoke Lemma 3.3 to deduce the result from Case III.

Proof of Theorem 1.4. The proof follows the preceding one. First of all, we have the analogue of Lemma 2.1.

Lemma 2.2. Let $C$ be a circle, let $F$ consist of finitely many subarcs of $C$ and let $I \subset F$ be a subarc of $F$. Suppose that there is an $\alpha<1$ for which the following is true: for every $\rho$ with $\rho(F)=0$ there are measures $\nu$ and $\mu$ such that $\nu$ is supported on $F$, it has log-convex density on $I, \mu$ is supported on $C, \mu(F)=0,\|\mu\| \leq \alpha\|\rho\|$ and $\operatorname{Bal}(\rho, F)=\nu+\operatorname{Bal}(\mu, F)$. Then for all measures $\rho$ on $C$ with $\rho(F)=0$ the density of $\operatorname{Bal}(\rho, F)$ is log-convex on $I$. 
The proof is the same as that of Lemma 2.1. Now we can follow the proof of Theorem 1.3.

Case I. F is an arc. Here we could simply refer to Lemma 4.9 in [2], where the log-convexity in question is proved, but for completeness we include a proof. We may assume that $C$ is the unit circle $C_{1}$, and let $I \subset C_{1}$ be an arc on it, say $I=J_{A}:=\left\{e^{i t} \mid t \notin(-A, A)\right\}$. We have to show that the density of the balayage of $\delta_{e^{i s}}$ with $s \in(-A, A)$ is log-convex on $I$, i.e., if $v\left(\delta_{e^{i s}} ; t\right)$ is this density at the point $e^{i t}$, then $v\left(\delta_{e^{i s}} ; \cdot\right)$ is a log-convex function on the interval $[A, 2 \pi-A]$. In what follows all arguments are understood modulo $2 \pi$.

The mapping $z \rightarrow w$ with

$$
w=i \frac{z+1}{z-1}
$$

maps $J_{A}$ onto $[-\cot A / 2, \cot A / 2]$ while $e^{i t}$ is mapped into $x=\cot t / 2$, and $e^{i s}$ is mapped into $\lambda:=\cot s / 2$ with $|\lambda|>\cot A / 2$. Since

$$
d x=-\frac{1}{\sin ^{2} t / 2} d t
$$

it follows from (2.1) and from the conformal invariance of harmonic measures that

$$
v\left(\delta_{e^{i s}} ; t\right)=\frac{1}{\pi} \frac{\sqrt{\lambda^{2}-\cot ^{2} A / 2}}{|\lambda-\cot t / 2| \sqrt{\cot ^{2} A / 2-\cot ^{2} t / 2}} \frac{1}{\sin ^{2} t / 2} .
$$

If we substitute here $\lambda=\cot s / 2$ and make use of the identities

$$
\cot \alpha \pm \cot \beta=\frac{\sin (\beta \pm \alpha)}{\sin \alpha \sin \beta},
$$

then we obtain for the density in question the expression

$$
\frac{1}{\pi} \frac{\sqrt{\sin \frac{A-s}{2} \sin \frac{A+s}{2}}}{\sqrt{\sin \frac{t-A}{2} \sin \frac{t+A}{2}}} \frac{1}{\sin \frac{|t-s|}{2}},
$$

which is clearly log-convex in $t$ on $[A, 2 \pi-A]$.

Case II. F consists of finitely many arcs. This case follows from the one arc case via Lemma 2.2 exactly as in Case II in the proof of Theorem 1.3.

Case III. $\overline{\mathbb{C}} \backslash F$ is regular with respect to the Dirichlet problem. Just apply the argument of Case III from the proof of Theorem 1.3.

Case IV. $F \subset C$ is arbitrary. Apply again the argument of Case IV from the proof of Theorem 1.3.

Proof of Corollary 1.6. We have to show that the density of $\operatorname{Bal}\left(\delta_{\lambda}, F\right)$ is convex on every subinterval $I$ of $F$. Let $\lambda=a+i b$, and assume e.g. that $b>0, a \geq 0$. If the downward cone with vertex at $\lambda$ and with side slopes $\pm \sqrt{3}$ does not contain 
an interior point of $F$, then we form the balayage of $\delta_{\lambda}$ onto $F$ in two steps: first take it onto the real line, and then onto $F$. When we take the balayage onto the real line then we take it out of the upper half plane for which the harmonic measure is the well known Poisson kernel on that half plane, so $\operatorname{Bal}\left(\delta_{\lambda}, \mathbb{R}\right)$ has density $b / \pi\left(b^{2}+(x-a)^{2}\right)$, which is convex on $I$ (note that the function $1 /\left(1+x^{2}\right)$, which appears in the density of $\operatorname{Bal}\left(\delta_{i}, \mathbb{R}\right)$, is convex on $(-\infty,-1 / \sqrt{3})$ and on $(1 / \sqrt{3}, \infty))$. Now the corollary follows, since when we balayage further the measure $\left.\operatorname{Bal}\left(\delta_{\lambda}, \mathbb{R}\right)\right|_{\mathbb{R} \backslash F}$ onto $F$, then the density is again convex on $I$ by Theorem 1.3. This argument takes care of the cases where $\lambda$ belongs to the rhomboids in Figure 1 or to the two infinite cones with vertices at \pm 1 and with side slopes $\pm \sqrt{3}$.

On the other hand, if $\lambda \notin H$ but the aforementioned cone with vertex at $\lambda$ contains an inner point of $F$ then necessarily $b \geq \sqrt{2}$, and in this case we take the balayage of $\delta_{\lambda}$ first onto the interval $[-1,1]$. By Lemma 3.4 , for $b \geq \sqrt{2}$ the density of $\operatorname{Bal}\left(\delta_{\lambda},[-1,1]\right)$ is log-convex on $(-1,1)$, and to get $\operatorname{Bal}\left(\delta_{\lambda}, F\right)$ we have to take a further balayage of $\left.\operatorname{Bal}\left(\delta_{\lambda},[-1,1]\right)\right|_{[-1,1] \backslash F}$ onto $F$, which has again log-convex density on $I$ by Theorem 1.3 .

For later reference let us mention that the first part of the proof demonstrates $\log$-convexity of the density of $\operatorname{Bal}\left(\delta_{\lambda},[-1,1]\right)$ for all $\lambda=a+i b$ with $|a| \geq|b|$ because the function $1 /\left(1+x^{2}\right)$, which appears in the density of $\operatorname{Bal}\left(\delta_{i}, \mathbb{R}\right)$, is $\log$-convex on $(-\infty,-1)$ and on $(1, \infty))$.

\section{Lemmas}

We are going to formulate our first three lemmas for the real line, but they are equally true on circles (with arcs replacing intervals and $C \backslash F$ replacing $\mathbb{R} \backslash F$ ) with the same proofs.

In what follows $\operatorname{Int}(I)$ denotes the (one-dimensional) interior of $I$, and regularity of a closed set $F$ means that $\overline{\mathbb{C}} \backslash F$ is regular with respect to the Dirichlet problem.

Lemma 3.1. Let $I \subset \mathbb{R}$ be an interval. Then the measures in

$$
\{\operatorname{Bal}(\rho, F): I \subset F \subset \mathbb{R},\|\rho\| \leq 1, \rho(F)=0\}
$$

are absolutely continuous on $I$ and they have uniformly equicontinuous densities on compact subsets of $\operatorname{Int}(I)$.

Proof. First we prove the claim when $F$ is an interval. Indeed, if $F=[a, b]$, then the density of $\operatorname{Bal}\left(\delta_{\lambda}, F\right)$ is given by (2.1), and this gives also the absolute continuity of this balayage measure. Now formula (2.1) shows that if $\left[\alpha^{\prime}, \beta^{\prime}\right] \subset(\alpha, \beta)$ are fixed, then the derivatives of the densities of all $\operatorname{Bal}\left(\delta_{\lambda},[a, b]\right)$ with $a \leq \alpha, \beta \leq b$, $\lambda \notin[a, b]$ are uniformly bounded on $\left[\alpha^{\prime}, \beta^{\prime}\right]$. Hence it follows (by integration with respect to $\rho$ ) that

$$
\{\operatorname{Bal}(\rho,[a, b]): I \subset[a, b],\|\rho\| \leq 1, \rho([a, b])=0\}
$$

have uniformly equicontinuous densities on compact subsets of $\operatorname{Int}(I)$. 
However, if $I \subset F$ is arbitrary, then

$$
\left.\operatorname{Bal}(\rho, F)\right|_{I}=\operatorname{Bal}(\rho, I)-\operatorname{Bal}\left(\left.\operatorname{Bal}(\rho, F)\right|_{F \backslash I}, I\right),
$$

and the lemma follows from the just established interval case.

Lemma 3.2. Let $I \subset \mathbb{R}$ be an interval, let $F, F_{n} n=1,2, \ldots$ be regular compact sets such that $F_{n+1} \subseteq F_{n}, I \subset F=\bigcap_{n} F_{n}$, and let $\left\{\rho_{n}\right\}$ be a sequence of measures on $\mathbb{R}$ such that $\rho_{n}\left(F_{n}\right)=0$ and $\rho_{n} \rightarrow \rho$ in the weak* topology to some $\rho$ with $\rho(F)=0$. Then $\operatorname{Bal}\left(\rho_{n}, F_{n}\right) \rightarrow \operatorname{Bal}(\rho, F)$ in the weak* topology, and the densities of $\operatorname{Bal}\left(\rho_{n}, F_{n}\right)$ tend to the density of $\operatorname{Bal}(\rho, F)$ uniformly on compact subsets of $\operatorname{Int}(I)$.

Here the weak* topology is understood on the set of continuous functions on $\overline{\mathbb{C}}=\mathbb{C} \cup\{\infty\}$. In particular, if $\rho_{n} \rightarrow \rho$ in this topology, then $\left\|\rho_{n}\right\| \rightarrow\|\rho\|$.

Proof. Let $\mathcal{N} \subset \mathbb{N}$ be arbitrary, and select a subsequence $\mathcal{N}^{\prime}$ of $\mathcal{N}$ such that as $n \rightarrow \infty, n \in \mathcal{N}^{\prime}$, we have $\operatorname{Bal}\left(\rho_{n}, F_{n}\right) \rightarrow \sigma$ for some measure $\sigma$. Since $\operatorname{Bal}\left(\rho_{n}, F_{n}\right)$ is supported on $F_{n}$, it follows that $\sigma$ is supported on $F$.

Let $U$ be a continuous function on $F$ and let $u$ be the solution of the Dirichlet problem in $\overline{\mathbb{C}} \backslash F$ with boundary function $U$. By the regularity of the domain $\overline{\mathbb{C}} \backslash F$ this $u$ (defined as $U$ on the boundary) is continuous on $\overline{\mathbb{C}}$, hence

$$
\begin{aligned}
\int U d \sigma & =\lim _{n \rightarrow \infty, n \in \mathcal{N}^{\prime}} \int u d \operatorname{Bal}\left(\rho_{n}, F_{n}\right)=\lim _{n \rightarrow \infty, n \in \mathcal{N}^{\prime}} \int u d \rho_{n} \\
& =\int u d \rho=\int U d \operatorname{Bal}(\rho, F),
\end{aligned}
$$

where the second and fourth equalities follow from (1.3). Since this is true for all continuous $U$ on $F$, we can conclude $\sigma=\operatorname{Bal}(\rho, F)$, and since this is true for any subsequence $\mathcal{N} \subset \mathbb{N}$, we can conclude that $\operatorname{Bal}\left(\rho_{n}, F\right) \rightarrow \operatorname{Bal}(\rho, F)$ for all $n \rightarrow \infty$ in the weak* topology.

Let $I^{\prime}$ be a closed subinterval of $\operatorname{Int}(I)$. If $v_{n}$ is the density of $\operatorname{Bal}\left(\rho_{n}, F_{n}\right)$, then it follows from Lemma 3.1 and from the Arzelà-Ascoli theorem (which we can apply to $\left\{v_{n}\right\}$ because of the equicontinuity expressed in Lemma 3.1 and because this sequence is clearly uniformly bounded on $I^{\prime}$, since the $\left\|\rho_{n}\right\|$ are bounded) that from any subsequence of $\left\{v_{n}\right\}_{n \in \mathcal{N}}$ we can select a uniformly convergent subsequence $\left\{v_{n}\right\}_{n \in \mathcal{N}^{\prime}}, v_{n} \rightarrow v$ uniformly on $I^{\prime}$ as $n \rightarrow \infty, n \in \mathcal{N}^{\prime}$. Let $f$ be a continuous function with compact support in $\operatorname{Int}\left(I^{\prime}\right)$. We have, as $n \rightarrow \infty, n \in \mathcal{N}^{\prime}$, the just proven

$$
\int f d \operatorname{Bal}\left(\rho_{n}, F_{n}\right) \rightarrow \int f d \operatorname{Bal}(\rho, F),
$$

and at the same time

$$
\int f d \operatorname{Bal}\left(\rho_{n}, F_{n}\right)=\int f v_{n} \rightarrow \int f v,
$$

so

$$
\int f d \operatorname{Bal}(\rho, F)=\int f v .
$$


Since this is true for every such $f$, it follows that

$$
\left.\operatorname{Bal}(\rho, F)\right|_{\operatorname{Int}\left(I^{\prime}\right)}=v(x) d x
$$

i.e., $v$ is the density of $\operatorname{Bal}(\rho, F)$ on $\operatorname{Int}\left(I^{\prime}\right)$. Since this is true for any subsequence $\mathcal{N} \subset \mathbb{N}$, we can finally conclude that the whole sequence $\left\{v_{n}\right\}$ converges to $v$ uniformly on compact subsets of $\operatorname{Int}\left(I^{\prime}\right)$, and this proves the claim.

Lemma 3.3. Let $I$ be an interval in the real line and let $I \subset F \subset \mathbb{R}$ be an arbitrary closed set. Let furthermore $F_{n}, n=1,2, \ldots$, be regular closed sets such that $I \subseteq F_{n} \subseteq F_{n+1} \subseteq F$, for all $n$, and $F \backslash \bigcup_{n=1}^{\infty} F_{n}$ is of zero logarithmic capacity. Then for any $\rho$ on $\mathbb{R}$ with $\rho(F)=0$ the densities of $\operatorname{Bal}\left(\rho, F_{n}\right)$ tend to the density of $\operatorname{Bal}(\rho, F)$ uniformly on compact subsets of $\operatorname{Int}(I)$.

Proof. Let $E$ be a closed subinterval of $\operatorname{Int}(I)$, and let $U$ and $u_{n}$, respectively, be the solutions of the Dirichlet problem in $\overline{\mathbb{C}} \backslash F$ and $\overline{\mathbb{C}} \backslash F_{n}$, with boundary values equal to 1 on $E$ and 0 elsewhere. Then (extending $U$ and $u_{n}$ to the boundary with these boundary values) $u_{n}$ is continuous on $\overline{\mathbb{C}}$ except at the two endpoints of $E$, and $U \leq u_{n+1} \leq u_{n}$ for all $n$. By Harnack's theorem, $\left\{u_{n}\right\}$ converges on compact subsets of $\overline{\mathbb{C}} \backslash F$ to a harmonic function $u$. We claim that $U=u$. The fact $U \leq u \leq 1$ is clear, so $u$ has boundary limit 1 at every point of $E$ since $U$ does also.

On the other hand, if $z \in F_{n} \backslash E$ for some $n$, then $u$ has zero boundary limit at $z$ (because $U \leq u \leq u_{n}$ on $\overline{\mathbb{C}} \backslash F$ ). Therefore, as quasi-every point of $F$ belongs to $\bigcup_{n} F_{n}$, we can see that $u$ has boundary limit 1 on $E$ and 0 quasi-everywhere on $F \backslash E$, hence it is the solution of the Dirichlet problem in $\overline{\mathbb{C}} \backslash F$ with these boundary values. This proves $u=U$.

Since for $\lambda \notin F$ we have

$$
U(\lambda)=\omega(\lambda, E ; \overline{\mathbb{C}} \backslash F)=\operatorname{Bal}\left(\delta_{\lambda}, F\right)(E)
$$

and

$$
u_{n}(\lambda)=\omega\left(\lambda, E ; \overline{\mathbb{C}} \backslash F_{n}\right)=\operatorname{Bal}\left(\delta_{\lambda}, F_{n}\right)(E),
$$

from (1.2) and from Lebesgue's monotone convergence theorem we can conclude that $\operatorname{Bal}\left(\rho, F_{n}\right)(E) \rightarrow \operatorname{Bal}(\rho, F)(E)$ as $n \rightarrow \infty$. This is true for all subintervals $E$ of $\operatorname{Int}(I)$, and then, in view of Lemma 3.1, the lemma easily follows.

Lemma 3.4. For $b^{2} \geq 2$, the balayage measure $\operatorname{Bal}\left(\delta_{a+i b},[-1,1]\right)$ is log-convex on $(-1,1)$ for any $a$.

We note that this is no longer true for $b^{2}<2$. Moreover, if $a=0$, then the $y$ in the following proof is $\infty$ and (3.3) takes the form

$$
\frac{d \operatorname{Bal}\left(\delta_{i b},[-1,1]\right)}{d x}=\frac{|b| \sqrt{|\lambda+1||\lambda-1|}}{\pi} \frac{1}{\sqrt{1-x^{2}}\left(x^{2}+b^{2}\right)}
$$


whose second derivative at 0 is

$$
\frac{b^{2}-2}{\pi|b|^{3} \sqrt{|\lambda+1||\lambda-1|}}
$$

so $\operatorname{Bal}\left(\delta_{i b},[-1,1]\right)$ is not even convex around the origin when $b^{2}<2$.

Proof. We first recall the formula for the equilibrium measure of an arc (see Example 11.1.4 in [7]). Let $0 \leq \alpha<\beta \leq 2 \pi$. Denote by $\left[e^{i \alpha}, e^{i \beta}\right]:=\left\{e^{i \theta} \mid \alpha \leq \theta \leq \beta\right\}$ the corresponding arc of the unit circle $\mathbb{T}:=\{z|| z \mid=1\}$. Let $\gamma=\pi+\frac{\alpha+\beta}{2}$, that is, $e^{i \gamma}$ is the midpoint of the complementary arc $\mathbb{T} \backslash\left[e^{i \alpha}, e^{i \beta}\right]$. The equilibrium measure of the arc is given as

$$
\mu_{[\alpha, \beta]}=\frac{\cos \left(\frac{\theta}{2}-\frac{\alpha+\beta}{4}\right) d \theta}{2 \pi \sqrt{\sin \left(\frac{\beta-\theta}{2}\right) \sin \left(\frac{\theta-\alpha}{2}\right)}}=\frac{\left|e^{i \gamma}-e^{i \theta}\right|}{2 \pi \sqrt{\left|e^{i \theta}-e^{i \alpha}\right|\left|e^{i \theta}-e^{i \beta}\right|}} d \theta, \quad \alpha \leq \theta \leq \beta,
$$

and in this last form the circle (with radius 1 ) need not be the unit circle as long as $d \theta$ denotes arc length on it.

Now, let us derive a formula for $\operatorname{Bal}\left(\delta_{\lambda},[-1,1]\right)$ for $\lambda=a+i b, b>0$. Take inversion with respect to the circle with center $\lambda$ and radius $R=\sqrt{2 b}$. The image of $\mathbb{R}$ is a circle $K$ of radius one, with $\lambda$ being its north pole (see Figure 2). Denote the images of -1 and 1 by $A$ and $B$ respectively. The image under inversion of the interval $[-1,1]$ is the arc $\widehat{A B}$. Let us consider the triangle with vertices $-1,1$ and $\lambda$, and let $l$ (resp. $y$ ) denote the intersection with $\mathbb{R}$ of the interior (resp. exterior) angular bisectors at $\lambda$. Observe that $y$ is the image under the inversion of the midpoint $C$ of the gap $K \backslash \widehat{A B}$. Denote by $z$ the intersection with $\mathbb{R}$ of the line through $\lambda$ that is perpendicular to the line connecting 0 and $\lambda$. We may assume $a \geq 0$, and then $|\lambda-1| \leq|\lambda+1|$, and, as a consequence, $0 \leq l<1<z \leq y$. Also, from similarity of triangles, we derive that $z=\left(a^{2}+b^{2}\right) / a$.

Let $T$ be the image in $K$ of a generic point $x \in \mathbb{R}$. The distance and measure conversion formulas are

$$
\begin{aligned}
|C-T| & =\frac{2 b|y-x|}{|\lambda-x||\lambda-y|} \\
\frac{1}{\sqrt{|A-T||B-T|}} & =\frac{|\lambda-x| \sqrt{|\lambda+1||\lambda-1|}}{2 b \sqrt{1-x^{2}}} \\
|d T| & =\frac{2 b d x}{|\lambda-x|^{2}} .
\end{aligned}
$$

Using (3.1) we can write the formula for the equilibrium measure of $\widehat{A B}$ as

$$
d \mu_{\widehat{A B}}=\frac{|T-C|}{2 \pi \sqrt{|T-A||T-B|}}|d T| .
$$

Since harmonic measures are conformally invariant and $\lambda$ is mapped into the point infinity under the above inversion, the balayage measure $\operatorname{Bal}\left(\delta_{\lambda},[-1,1]\right)$ is the 


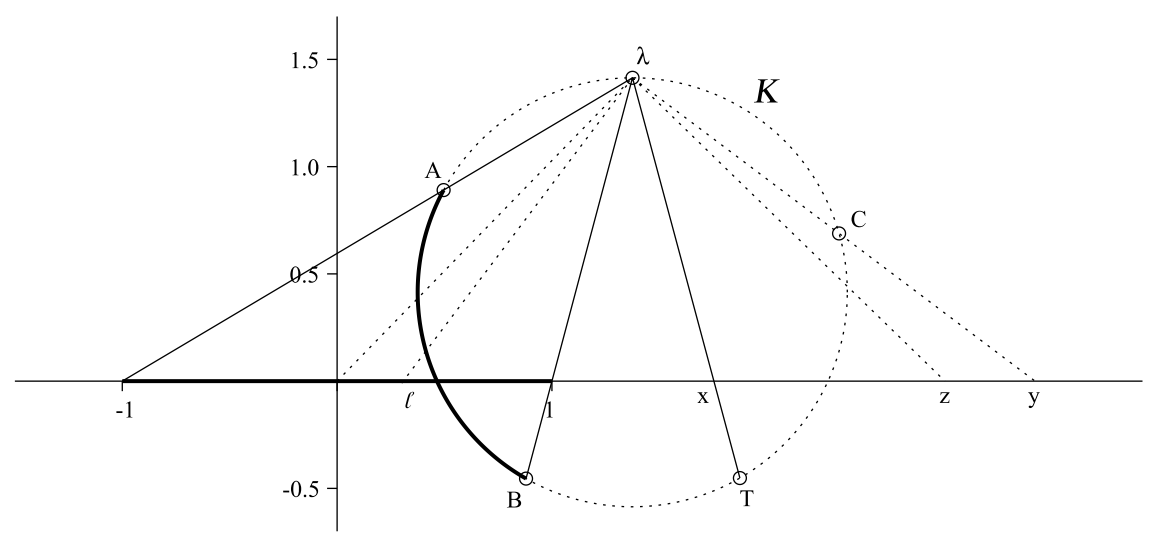

Figure 2. Balayage of $\lambda=a+i b$ onto $[-1,1]$.

transform of the equilibrium measure for the arc $\widehat{A B}$. Substituting the preceding values in (3.2) we obtain

$$
\frac{d \operatorname{Bal}\left(\delta_{\lambda},[-1,1]\right)}{d x}=\frac{b \sqrt{|\lambda+1||\lambda-1|}|y-x|}{\pi|\lambda-y| \sqrt{1-x^{2}}\left((x-a)^{2}+b^{2}\right)}=: \phi(x), \quad x \in[-1,1]
$$

which is the needed formula.

In proving log-convexity of the density we may assume, without loss of generality, that in $\lambda=a+i b$ we have $a \geq 0, b>0$. Let us first prove the lemma when $b=\sqrt{2}$. If $a \geq \sqrt{2}$ the $\log$-convexity in question follows from the last paragraph of the proof of Corollary 1.6, so in what follows let $a \leq \sqrt{2}$. Differentiating $\ln \phi(x)$ twice we get that

$$
g(x ; a, \sqrt{2}):=(\ln \phi(x))^{\prime \prime}=\frac{1+x^{2}}{\left(1-x^{2}\right)^{2}}+\frac{2(x-a)^{2}-4}{\left((x-a)^{2}+2\right)^{2}}-\frac{1}{(y-x)^{2}} .
$$

Observe that for $0 \leq a \leq \sqrt{2}$ and $x \in[-1,1]$ we have

$$
g(-|x| ; a, \sqrt{2}) \geq g(|x| ; a, \sqrt{2}) .
$$

Indeed, $(y-2) /\left(y^{2}+2\right)^{2}$ is an increasing function on the interval $[0,6]$, and $0 \leq$ $(|x|-a)^{2} \leq(|x|+a)^{2}<6$ in this case. Hence, we may assume $x \in[0,1]$. From 
$y \geq z=\left(a^{2}+b^{2}\right) / a$ and (3.4) we conclude that

$$
\begin{aligned}
g(x ; a, \sqrt{2}) & \geq \frac{1+x^{2}}{\left(1-x^{2}\right)^{2}}+\frac{2(x-a)^{2}-4}{\left((x-a)^{2}+2\right)^{2}}-\frac{1}{\left(\frac{a^{2}+2}{a}-x\right)^{2}} \\
& =\frac{1+x^{2}}{\left(1-x^{2}\right)^{2}}-1+\frac{(x-a)^{4}+6(x-a)^{2}}{\left((x-a)^{2}+2\right)^{2}}-\frac{1}{\left(\frac{a^{2}+2}{a}-x\right)^{2}} \\
& \geq \frac{x^{2}\left(3-x^{2}\right)}{\left(1-x^{2}\right)^{2}}+\frac{6(x-a)^{2}}{\left((x-a)^{2}+2\right)^{2}}-\frac{a^{2}}{(a(a-x)+2)^{2}} \\
& =: U(x, a) .
\end{aligned}
$$

If $0 \leq x \leq a / 2$, then

$$
U(x, a) \geq \frac{6(x-a)^{2}-a^{2}}{\left((x-a)^{2}+2\right)^{2}} \geq \frac{a^{2}}{2\left((x-a)^{2}+2\right)^{2}} \geq 0,
$$

and if $a / 2<x \leq 1$, then

$$
U(x, a) \geq \frac{a^{2}\left(3-x^{2}\right)}{4\left(1-x^{2}\right)^{2}}-\frac{a^{2}}{\left(a^{2}-a+2\right)^{2}} \geq \frac{3 a^{2}}{4}-\frac{16 a^{2}}{49} \geq 0 .
$$

This establishes the lemma when $b=\sqrt{2}$.

If $b>\sqrt{2}$, then we first balayage $\delta_{\lambda}$ onto the line $\Im m(z)=\sqrt{2}$ (notice that this leaves the potential on the real line unchanged up to a constant), then take the balayage of the resulting measure onto $[-1,1]$ and use the superposition principle $(1.2)$ and the just verified case when $b=\sqrt{2}$.

\section{References}

[1] Ancona, A.: Démonstration d'une conjecture sur la capacité et l'effilement. C. $R$. Acad. Sci. Paris Sèr. I Math. 297 (1983), no. 7, 393-395.

[2] Benko, D., Damelin, S. B. and Dragnev, P. D.: On supports of equilibrium measures with concave signed equilibria. J. Comput. Anal. Appl. 14 (2012), no. 4, $752-766$.

[3] Benko, D. And Dragnev, P. D.: Balayage ping pong: A convexity of equilibrium measures. Constr. Approx. 36 (2012), no. 2, 191-214.

[4] Garnett, J. B. And Marshall, D. E.: Harmonic measure. New Mathematical Monographs 2, Cambridge University Press, Cambridge, 2005.

[5] Landkof, N. S.: Foundations of modern potential theory. Grundlehren der mathematischen Wissenschaften 180, Springer-Verlag, New York-Heidelberg, 1972.

[6] Ransford, T.: Potential theory in the complex plane. Cambridge University Press, Cambridge, 1995.

[7] Simon, B.: Orthogonal polynomials on the unit circle, Part 2, Spectral theory. American Mathematical Society Colloquium Publications 54, part 2, American Mathematical Society, Providence, RI, 2005. 
[8] SAfF, E. B. And Totik, V.: Logarithmic potentials with external fields. Grundlehren der mathematischen Wissenschaften 316, Springer Verlag, Berlin-Heidelberg, 1997.

[9] Tотік, V.: Polynomial inverse images and polynomial inequalities. Acta Math. 187 (2001), no. 1, 139-160.

Received October 31, 2010.

DAvid Benko: Department of Mathematics and Statistics, University of South Alabama, ILB 325, Mobile, AL 36688, USA.

E-mail: dbenko@jaguar1.usouthal.edu

Peter Dragnev: Department of Mathematical Sciences, Indiana University-Purdue University Fort Wayne, Fort Wayne, IN 46805, USA.

E-mail: dragnevp@ipfw.edu

Vilmos Tотік: Analysis and Stochastics Research Group, Bolyai Institute, University of Szeged, Szeged, Aradi v. tere 1, 6720, Hungary;

and

Department of Mathematics and Statistics, University of South Florida, 4202 E. Fowler Ave, PHY 114, Tampa, FL 33620-5700, USA.

E-mail: totik@mail.usf.edu 